\title{
Characterizations of Strong Strictly Singular Operators
}

\author{
C. Ganesa Moorthy ${ }^{1}$ and C. T. Ramasamy ${ }^{2}$ \\ ${ }^{1}$ Department of Mathematics, Alagappa University, Karaikudi 630 005, India \\ ${ }^{2}$ Department of Mathematics, H. H. The Rajah's College, Pudukkottai, Tamil Nadu 622001, India
}

Correspondence should be addressed to C. T. Ramasamy; ctrams83@gmail.com

Received 26 August 2013; Accepted 12 October 2013

Academic Editors: W. Klingenberg, X. Tang, and C. Yin

Copyright (C) 2013 C. Ganesa Moorthy and C. T. Ramasamy. This is an open access article distributed under the Creative Commons Attribution License, which permits unrestricted use, distribution, and reproduction in any medium, provided the original work is properly cited.

\begin{abstract}
A new class of operators called strong strictly singular operators on normed spaces is introduced. This class includes the class of precompact operators, and is contained in the class of strictly singular operators. Some properties and characterizations for these operators are derived.
\end{abstract}

\section{Introduction}

The spaces $X$ and $Y$ will denote normed spaces, and $T: X \rightarrow$ $Y$ will denote a bounded linear mapping from a normed space $X$ into a normed space $Y$ in this paper. Completeness is assumed only when it is specifically stated. An operator $T$ is called strictly singular if it does not have a bounded inverse on any infinite dimensional subspace contained in $X$. If $T\left(B_{X}\right)$ is totally bounded in $Y$, where $B_{X}$ is the open unit ball in $X$, then $T$ is called a precompact operator. If $\overline{T\left(B_{X}\right)}$, closure of $T\left(B_{X}\right)$, is compact in $Y$, then $T$ is called a compact operator. Every precompact operator is strictly singular (cf: [1]). The collection of all strictly singular (precompact) operators from $X$ into $Y$ forms a closed subspace of the normed space $\mathscr{B}(X, Y)$, the collection of all bounded linear operators from $X$ into $Y$ (cf: [1]). The collection of strictly singular (precompact) operators on $X$ (from $X$ into $X$ ) forms a closed ideal of the normed algebra $\mathscr{B}(X)(=\mathscr{B}(X, X))$ (cf: [1]). A linear transformation $T: X \rightarrow Y$ has a bounded inverse if and only if $\|x\| \leq c\|T x\|$ for all $x \in X$, for some $c>0$. It is easy to see as a consequence of the open mapping theorem that a continuous linear transformation $T$ from a Banach space $X$ into a Banach space $Y$ has closed range, if and only if for given $x \in X$, there is an element $y \in X$ such that Tx $=T y$ and $\|y\| \leq c\|T y\|$, for some fixed $c>0$ (see [2]). This gives a motivation to define a new class of operators called strong strictly singular operators.
Write $\widehat{X}=X / N(T)$, where $N(T)$ is the null space of $T$, as the quotient space endowed with the quotient norm. Let $\pi_{T}$ denote the quotient map from $X$ onto $\widehat{X}$. The 1-1 operator $\widehat{T}$ : $\widehat{X} \rightarrow Y$ induced by $T$ is defined by $\widehat{T}\left(x+N(T)=\pi_{T}(x)\right)=$ $T x$. Note that $\widehat{T}$ is $1-1$ and linear with range same as the range of $T$. If $T$ is $1-1$ then $T=\widehat{T}$. Also, $B_{\widehat{X}}=\pi_{T}\left(B_{X}\right)$ and $T\left(B_{X}\right)=$ $\widehat{T}\left(B_{\widehat{X}}\right)$. So, we have the following conclusions.

(a) $T$ is precompact on $X$ if and only if $\widehat{T}$ is precompact on $\widehat{X}$.

(b) $T$ is compact on $X$ if and only if $\widehat{T}$ is compact on $\widehat{X}$.

\section{Definition}

Definition 1. An operator $T: X \rightarrow Y$ is said to be strong strictly singular if for any subspace $M$ of $X$ such that dim $T(M)=\infty$ and the null space $N(T) \subset M$ there is no positive number $c$ with the property: for a given $x \in M$, there is an element $y \in M$ such that $T x=T y$ and $\|y\| \leq c\|T y\|$.

Lemma 2. Every strong strictly singular operator is strictly singular.

Proof. Let $T$ be strong strictly singular. Suppose $M_{0}$ is an infinite dimensional subspace of $X$ such that the restriction of $T$ on $M_{0}$ has a bounded inverse. Then, there is a positive constant $c$ such that $\|x\| \leq c\|T x\|$ for every $x \in M_{0}$. Since $T$ 
is 1-1 on $M_{0}$, we have $N(T) \cap M_{0}=\{0\}$. Let us consider that $M=M_{0}+N(T)$. Then, $\operatorname{dim} T(M)=\operatorname{dim} T\left(M_{0}\right)=\infty$. Also, for given $x \in M$, there are $y \in M_{0}$ and $z \in N(T)$ such that $x=y+z$ and $\|y\| \leq c\|T y\|=c\|T x\|$. This shows that $T$ is not strong strictly singular. This contradiction shows that $T$ is strictly singular, whenever $T$ is strong strictly singular.

The converse of Lemma 2 is not true. For example, there is an onto continuous linear operator $T: \ell_{1} \rightarrow \ell_{2}$ which is strictly singular [1, page 89 , III.3.7] but not strong strictly singular, because it has a closed range.

A simple characterization for strong strictly singularity is the following lemma.

Lemma 3. $T$ is strong strictly singular if and only if $\widehat{T}$ is strictly singular.

Proof. Suppose that $\widehat{M}_{0}$ is any subspace of $\widehat{X}$ such that, for all $x+N(T) \in \widehat{M}_{0}$, we have $\|x+N(T)\| \leq c\|\widehat{T}(x+N(T))\|$, for some fixed $c>0$. Then, for any fixed $c^{\prime}>c$, for given $x \in \pi_{T}^{-1}\left(\widehat{M}_{o}\right)$, there is an element $y \in \pi_{T}^{-1}\left(\widehat{M}_{0}\right)$ such that $x+N(T)=y+N(T)$ and

$$
\begin{aligned}
\|y\| & \leq\left(\frac{c^{\prime}}{c}\right)\|x+N\| \leq c^{\prime}\|\widehat{T}(x+N(T))\| \\
& =c^{\prime}\|\widehat{T}(y+N(T))\|=c^{\prime}\|T(y)\|, \quad T x=T y .
\end{aligned}
$$

This proves that if $T$ is strong strictly singular, then $\widehat{T}$ is strictly singular.

On the other hand, suppose that $M$ is any subspace of $X$ such that $N(T) \subset M$ and such that for a given $x \in M$ there is an element $y \in M$ such that $T x=T y$ and $\|y\| \leq c\|T y\|$, for fixed $c>0$. Then

$$
\begin{aligned}
\|x+N(T)\| \leq & \|y\| \leq c\|T y\|=c\|\widehat{T}(y+N(T))\| \\
= & c\|\widehat{T}(x+N(T))\|, \\
& \quad \text { for any } x+N(T) \in \pi_{T}(M) .
\end{aligned}
$$

This proves that if $\widehat{T}$ is strictly singular, then $T$ is strong strictly singular. This completes the proof.

Corollary 4. If $T$ is 1-1 and strictly singular, then $T$ is strong strictly singular.

Corollary 5. Every precompact operator is strong strictly singular.

Proof. If $T: X \rightarrow Y$ is precompact, then $\widehat{T}$ so is. Hence $\widehat{T}$ is strictly singular, and hence $T$ is strong strictly singular.

Note that the inclusion map $I: \ell_{p} \rightarrow \ell_{q}, 1 \leq p<q<\infty$, is strong strictly singular but not precompact [3, page 170].

Although the converse of Lemma 2 is not true in general, it is true partially which is seen from the next theorem.

Theorem 6. Let $T: X \rightarrow Y$ be a strictly singular operator. Suppose that $X=N(T) \oplus Z$, for some subspace $Z$ of $X$, and the corresponding projection $P: X \rightarrow Z$ defined by $p(y+z)=$ $z$ with $y \in N(T)$ and $z \in Z$ is continuous. Then $T$ is strong strictly singular.

Proof. Let us consider a subspace $M$ of $X$ such that $N(T) \subset$ $M$ and such that for each given $x \in X$, there is a $y \in M$ satisfying $T x=T y$ and $\|y\| \leq c\|T x\|$, for some fixed $c>0$. Let $M_{0}=P(M)$. Then $T$ is 1-1 on $M_{0}$. Moreover, for given $x \in M_{0}$, there are $y_{0} \in M$ and $z_{0} \in N(T)$ such that $y_{0}=x+z_{0}$, and $T x=T y_{0}$. For this $y_{0} \in M$, there is a $y \in M$ such that $\|y\| \leq$ $c\left\|T y_{0}\right\|$ and $T y=T y_{0}$. For this $y \in M$, let us write $y=x+z_{1}$, with $z_{1} \in N(T)$. Then, we have $\|x\|=\|P y\| \leq\|P\|\|y\| \leq$ $c\|P\|\left\|T y_{0}\right\|=c\|P\|\|T x\|$. Thus, we have $\|x\| \leq c\|P\|\|T x\|$ for every $x \in M_{0}$. Since $T$ is strictly singular, $\operatorname{dim} M_{0}<\infty$. So, $\operatorname{dim} T(M)=\operatorname{dim} M_{0}<\infty$. This proves that $T$ is a strong strictly singular operator.

Corollary 7. Let $T: X \rightarrow Y$ be a strictly singular operator on a Banach space $X$ such that $X=N(T) \oplus Z$, for some closed subspace $Z$ of $X$. Then $T$ is strong strictly singular.

Corollary 8. Let $T: X \rightarrow Y$ be a strictly singular operator such that $\operatorname{dim}(N(T))<\infty$. Then $T$ is strong strictly singular.

\section{Characterization}

A known classical characterization of strictly singular operators is given in Theorem 9. A new similar characterization for strictly singular operators is found in the paper [4], when the operators are 1-1 and they are from a Banach space $X$ into $X$ itself.

Theorem 9 (cf: [1]). Suppose $T \in \mathscr{B}(X, Y)$. The following four statements are equivalent.

(i) $T$ is strictly singular.

(ii) For every infinite dimensional subspace $M \subset X$, there exists an infinite dimensional subspace $N \subset M$ such that $T$ is precompact on $N$.

(iii) Given $\epsilon>0$ and given $M$, an infinite dimensional subspace of $X$, there exists an infinite dimensional subspace $N \subset M$ such that $T$ restricted to $N$ has norm not exceeding $\epsilon$.

(iv) Given $\epsilon>0$ and given $M$, an infinite dimensional subspace of $X$, there exists an infinite dimensional subspace $N \subset M$ such that $T$ restricted to $N$ is precompact and it has norm not exceeding $\epsilon$.

Using Lemma 3 and Theorem 9, one can easily verify the following.

Theorem 10. Suppose $T \in \mathscr{B}(X, Y)$. The following four statements are equivalent.

(i) $T$ is strong strictly singular.

(ii) For every infinite dimensional subspace $M \subset X$ satisfying $N(T) \subset M$ and $\operatorname{dim} T(M)=\infty$, there exists an infinite dimensional subspace $N$ of $X$ satisfying 
$N(T) \subset N \subset M$ and $\operatorname{dim} T(N)=\infty$ such that $T$ is precompact on $N$.

(iii) Given $\epsilon>0$ and given $M$, an infinite dimensional subspace of $X$, satisfying $N(T) \subset M$ and $\operatorname{dim} T(M)=$ $\infty$, there exists an infinite dimensional subspace $N$ of $X$ satisfying $N(T) \subset N \subset M$ and $\operatorname{dim} T(N)=\infty$ such that $T$ restricted to $N$ has norm not exceeding $\epsilon$.

(iv) Given $\epsilon>0$ and given $M$, an infinite dimensional subspace of $X$ satisfying $N(T) \subset M$ and $\operatorname{dim} T(M)=$ $\infty$, there exists an infinite dimensional subspace $N$ of $X$ satisfying $N(T) \subset N \subset M$ and $\operatorname{dim} T(N)=\infty$ such that $T$ restricted to $N$ is precompact and its has norm not exceeding $\epsilon$.

The following interesting theorem on automatic continuity is due to van Dulst [5].

Theorem 11 (see [5]). Let $T: X \rightarrow Y$ be a linear transformation (need not be continuous). Suppose that there exists a constant $c>0$ with the property that every infinity dimensional subspace of $X$ contains a vector $x$ such that $\|x\|=$ 1 and $\|T x\|<c$. Then there exists a subspace $L \subset X$ with $\operatorname{dim} X / L<\infty$ such that $T_{L}$, the restriction of $T$ to $L$, is continuous.

Corollary 12. Let $T: X \rightarrow Y$ be a bounded linear transformation. Then the following statements are equivalent.

(a) $T$ is strong strictly singular.

(b) For a given subspace $M \subset X$ with $\operatorname{dim} T(M)=\infty$, there is no positive constant c such that for any subspace $M_{1}$ of $M$ with $\operatorname{dim} T\left(M_{1}\right)=\infty$, there is an element $x \in M_{1}+N(T)$ with $\|x\| \leq c\|T x\|$.

Proof. (a) $\Rightarrow$ (b): Suppose that (b) fails to be true. Then there is a subspace $M$ of $X$ satisfying $\operatorname{dim} T(M)=\infty$ and there is a positive constant $c$ such that for every subspace $M_{1}$ of $M$ satisfying $\operatorname{dim} T\left(M_{1}\right)=\infty$ there is an element $x \in M_{1}+N(T)$ such that $\|x\| \leq c\|T x\|$. Then, there is a subspace $\widehat{M}_{0}$ of $\pi_{T}(M)$ such that $\operatorname{dim} \pi_{T}(M) / \widehat{M}_{0}<\infty$ and $\widehat{T}$ restricted to $\widehat{M}_{0}$ is a homeomorphism; which follows from Theorem 11. So, $\widehat{T}$ is not strictly singular. Hence, $T$ is not strong strictly singular. This proves (a) $\Rightarrow$ (b).

(b) $\Rightarrow$ (a): Trivial.

\section{Properties}

Proposition 13. Let $T_{n}: X \rightarrow Y$ be a sequence of strong strictly singular operators such that $\left\|T_{n}-T\right\| \rightarrow 0$ for some operator $T: X \rightarrow Y$. Suppose that $N(T)=N\left(T_{i}\right)=N\left(T_{j}\right)$, for all $i, j$. Then $T$ is also strong strictly singular.

Proof. Note that $\left\|\widehat{T}_{n}-\widehat{T}\right\| \rightarrow 0$ and each $\widehat{T}_{n}$ is strictly singular, by Lemma 3 . So $\widehat{T}$ is strictly singular. Hence $T$ is strong strictly singular, by Lemma 3 .

The following technical lemma is on vector spaces.
Lemma 14. Let $M$ be an infinite dimensional subspace of $X \times$ $Y$, where $X$ and $Y$ are vector spaces over the same field. Then at least one of the following is true.

(i) There is an infinite dimensional subspace $X_{1}$ of $X$ such that $X_{1} \times\{0\} \subset M$.

(ii) There is an infinite dimensional subspace $Y_{1}$ of $Y$ such that $\{0\} \times Y_{1} \subset M$.

(iii) There are infinite dimensional subspaces $X_{1}$ and $Y_{1}$ of $X$ and $Y$, respectively, and there is an onto isomorphism $L: X_{1} \rightarrow Y_{1}$ such that $\left\{(x, L x): x \in X_{1}\right\} \subset M$.

Proof. Let $M_{X}, M_{Y}$ be the projections of $M$ on $X$ and $Y$, respectively. Then $M_{X}$ is of infinite dimension, or $M_{Y}$ is of infinite dimension. Suppose that $M_{X}$ is of infinite dimension. Let $S_{X}$ be a basis of $M_{X}$. To each $x \in S_{X}$, find an element $L x \in$ $M_{Y}$ such that $(x, L x) \in M$. Extend the map $x \mapsto L x$ to $L$ : $M_{X} \rightarrow M_{Y}$ as a linear mapping. If $\operatorname{dim} L\left(M_{X}\right)<\infty$, then we select $X_{1}$ as $\operatorname{ker} L$ so that (i) is true. If $\operatorname{dim} L\left(M_{X}\right)=\infty$, then find a subspace $X_{1}$ of $M_{X}$ such that $L\left(X_{1}\right)=L\left(M_{X}\right)$ and $L$ is 1-1 on $X_{1}$. In this case (iii) is true with $Y_{1}=L\left(M_{X}\right)$. Similarly, we can show that either (ii) or (iii) is true if $\operatorname{dim} M_{Y}=\infty$.

Remark 15. It is possible to select $X_{1}$ and $Y_{1}$ such that cardinalities of bases for $X_{1}$ and $Y_{1}$ are equal to the cardinality of a basis for $M$.

Theorem 16. Let $T_{1}: X_{1} \rightarrow Y_{1}, T_{2}: X_{2} \rightarrow Y_{2}$ be bounded linear operators between normed spaces. Define a bounded linear operator $T=T_{1} \times T_{2}: X_{1} \times X_{2} \rightarrow Y_{1} \times Y_{2}$ by $T\left(x_{1}, x_{2}\right)=\left(T_{1} x_{1}, T_{2} x_{2}\right)$. Then one has the following.

(i) If $T_{1}$ and $T_{2}$ are precompact (compact), then $T$ is precompact (compact).

(ii) If $T_{1}$ and $T_{2}$ are strictly singular operators, then $T$ is a strictly singular operator.

(iii) If $T_{1}$ and $T_{2}$ are strong strictly singular operators, then $T$ is a strong strictly singular operator.

Proof. (i) Is obvious. To prove (ii), suppose that $T_{1}$ and $T_{2}$ are strictly singular operators. Let $M$ be infinite dimensional subspace of $X_{1} \times X_{2}$. If there is an infinite dimensional subspace $X_{1}^{\prime}$ of $X_{1}$ such that $X_{1}^{\prime} \times\{0\} \subset M$, then we can find an infinite dimensional subspace $X_{1}^{\prime \prime}$ of $X_{1}^{\prime}$ such that $T_{1}$ is precompact on $X_{1}^{\prime \prime}$. In this case, there is an infinite dimensional subspace $X_{1}^{\prime \prime} \times\{0\}$ of $M$ such that $T$ is precompact on $X_{1}^{\prime \prime} \times\{0\}$. Similarly, if there is an infinite dimensional subspace $X_{2}^{\prime}$ of $X_{2}$ such that $\{0\} \times X_{2}^{\prime} \subset M$, then $T$ is precompact on an infinite dimensional subspace of $M$. Suppose that there are infinite dimensional subspaces $X_{1}^{\prime}$ and $X_{2}$ of $X_{1}$ and $X_{2}$, respectively, and an onto linear isomorphism $L: X_{1}^{\prime} \rightarrow X_{2}^{\prime}$ such that $\left\{\left(x_{1}, L x_{1}\right): x_{1} \in X_{1}^{\prime}\right\} \subset$ $M$. Find an infinite dimensional subspace $X_{1}^{\prime \prime}$ of $X_{1}^{\prime}$ such that $T_{1}$ is precompact on $X_{1}^{\prime \prime}$. Find an infinite dimensional subspace $X_{2}^{\prime \prime}$ of $L\left(X_{1}^{\prime \prime}\right)$ such that $T_{2}$ is precompact on $X_{2}^{\prime \prime}$. Then, $T$ is precompact on an infinite dimensional subspace $L^{-1}\left(X_{2}^{\prime \prime}\right) \times X_{2}^{\prime \prime}$ of $M$. This proves (ii). To prove (iii), suppose that $T_{1}$ and $T_{2}$ are strong strictly singular operators. Note that 
$N(T)=N\left(T_{1}\right) \times N\left(T_{2}\right)$ and $\left(X_{1} \times X_{2}\right) / N(T)=X_{1} / N\left(T_{1}\right) \times$ $X_{2} / N\left(T_{2}\right)$. So the natural mapping from $\left(X_{1} \times X_{2}\right) / N(T)$ to $Y_{1} \times Y_{2}$ coincides with the natural mapping from $X_{1} / N\left(T_{1}\right) \times$ $X_{2} / N\left(T_{2}\right)$ to $Y_{1} \times Y_{2}$. Since, by (ii), the later is strictly singular and the former is also strictly singular. So, $T$ is strong strictly singular. This proves (iii).

The following corollary is well known.

Corollary 17. If $T_{1}: X \rightarrow Y, T_{2}: X \rightarrow Y$ are two strictly singular (precompact) operators. Then $T_{1}+T_{2}$ is strictly singular (precompact).

Proof. It is follows from the continuous mappings $x \mapsto(x, x)$ from $X$ into $X \times X,\left(y_{1}, y_{2}\right) \mapsto y_{1}+y_{2}$ from $Y \times Y$ into $Y$ and strictly singular (precompact) mapping $\left(x_{1}, x_{2}\right) \mapsto$ $\left(T_{1} x_{1}, T_{2} x_{2}\right)$ from $X \times X$ into $Y \times Y$.

Lemma 18. Let $T: X \rightarrow Y$ be a strong strictly singular operator. Let $S: Y \rightarrow Z$ be a 1-1 continuous operator. Then $S \circ T$ is strong strictly singular.

Proof. Note that $N(T)=N(S \circ T)$. Since the natural mapping $S_{1}: X / N(T) \rightarrow Z$ is strictly singular, the mapping $S_{1}:$ $X / N(S \circ T) \rightarrow Z$ is strictly singular. Then, by Lemma $3, S \circ T$ is strong strictly singular.

Corollary 17, Lemma 18, and Proposition 13 lead to natural questions about limits of sequences of strong strictly singular operators and sum of two strong strictly singular operators.

\section{References}

[1] S. Goldberg, Unbounded Linear Operators, Theory and Applications, McGraw-Hill, New York, NY, USA, 1966.

[2] C. G. Moorthy and P. S. Johnson, "Closed range multiplication operators," in Mathematical Analysis and Applications, S. Nanda and G. P. Rajasekar, Eds., pp. 133-138, Narosa Pubilications, 2004.

[3] Y. A. Abramovich and C. D. Aliprantis, An Invitation to Operator Theory, vol. 60 of Graduate Studies in Mathematics, AMS, 2002.

[4] G. Androulakis and P. Enflo, "A property of strictly singular oneto-one operators," Arkiv for Matematik, vol. 41, no. 2, pp. 233252, 2003.

[5] D. van Dulst, "On strictly singular operators," Compositio Mathematica, vol. 23, no. 2, pp. 169-183, 1971. 


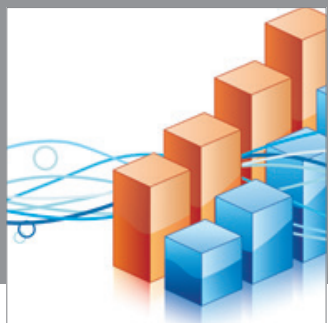

Advances in

Operations Research

mansans

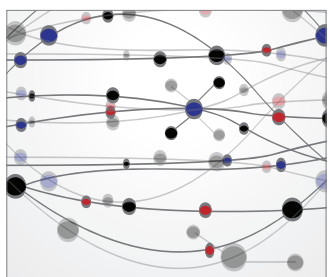

The Scientific World Journal
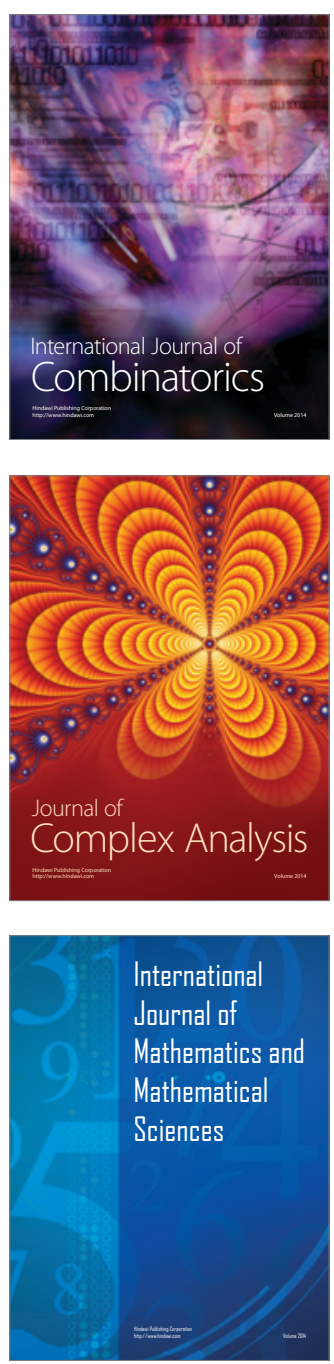
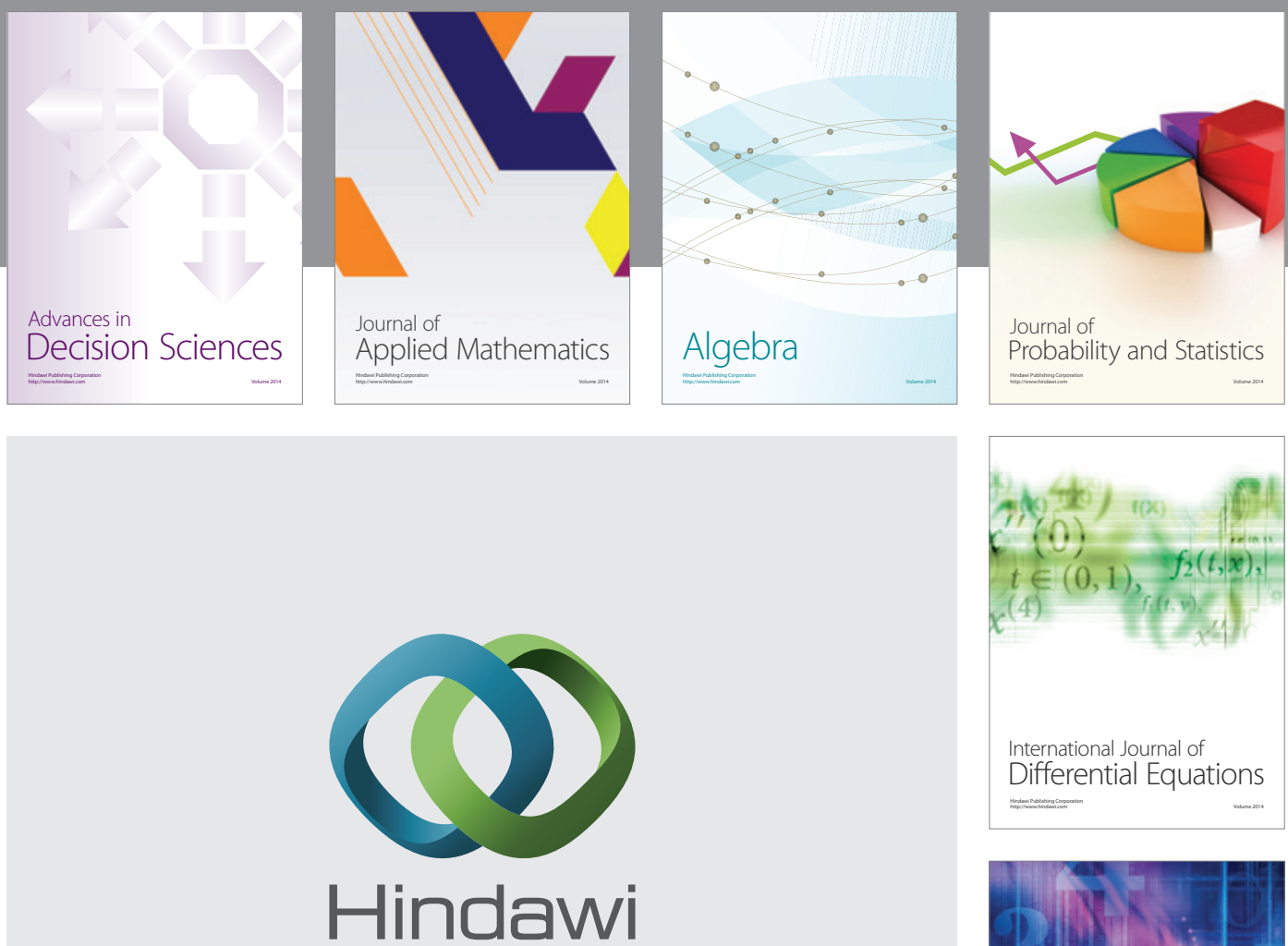

Submit your manuscripts at http://www.hindawi.com
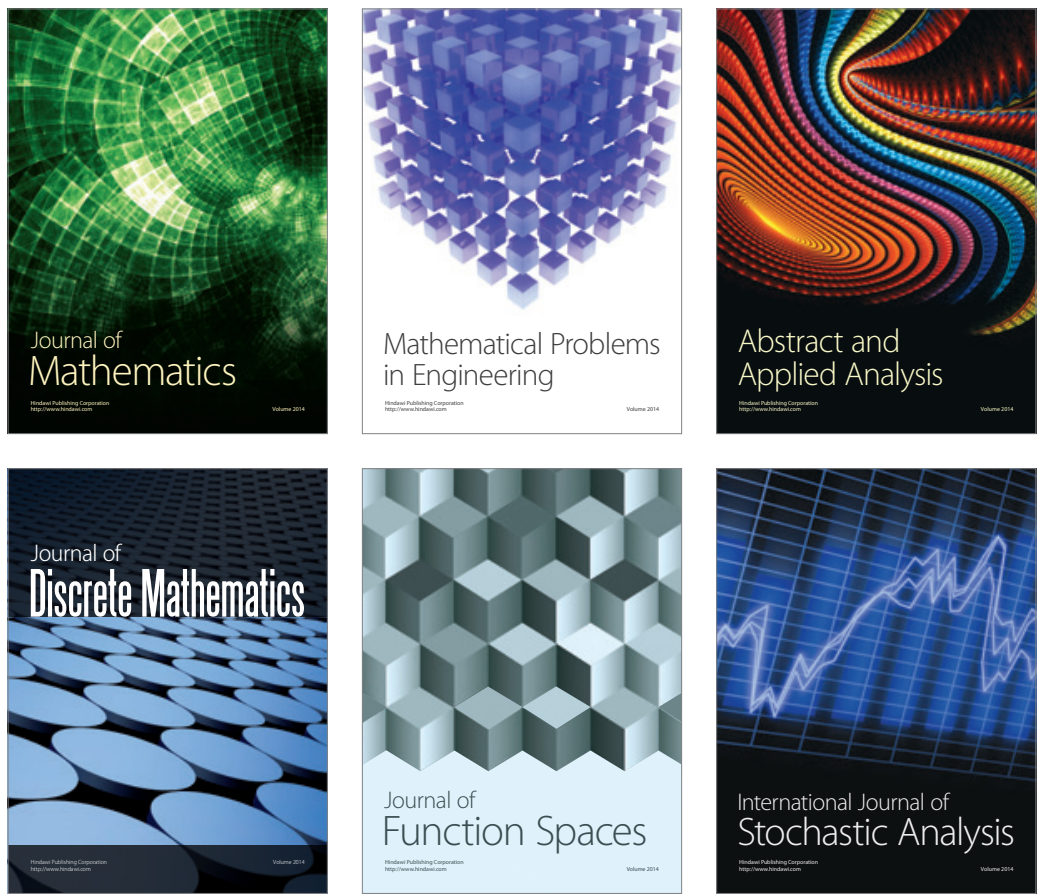

Journal of

Function Spaces

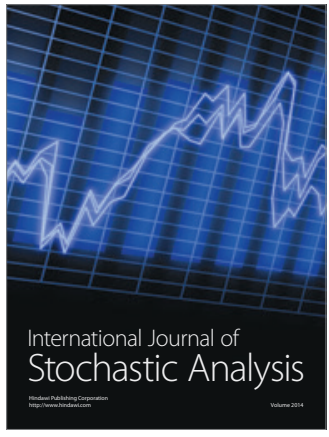

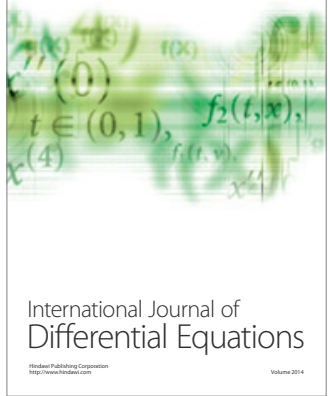
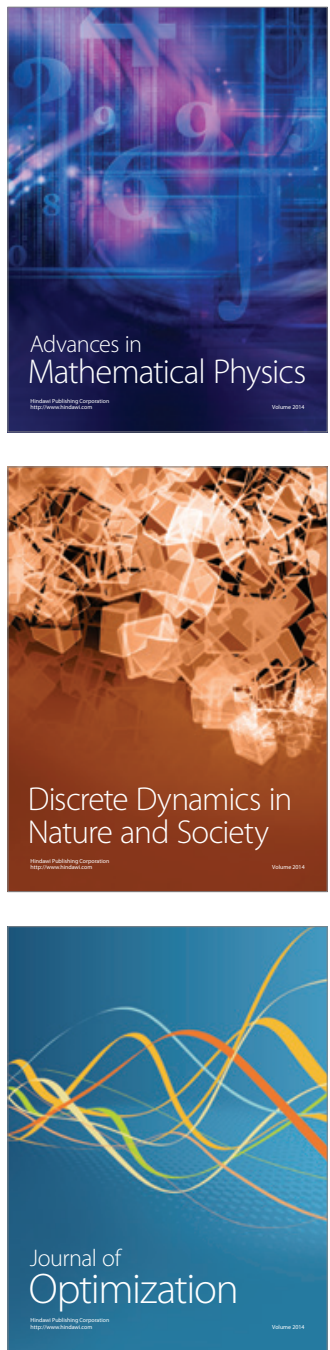